\title{
Transaction Decision Making for Intelligent Distributed Units based on Blockchain as a Service (BaaS)
}

\author{
Xiangxiang Xiao ${ }^{\mathrm{a}, \mathrm{b},{ }^{*}}$, Bin Duan ${ }^{\mathrm{a}, \mathrm{b}}$, Jun Lai ${ }^{\mathrm{a}}$, and Tao $\mathrm{Li}^{\mathrm{a}}$ \\ ${ }^{a}$ College of Information Engineering, Xiangtan University, Xiangtan, 411100, China \\ ${ }^{b}$ Collaborative Innovation Center of Wind Power Equipment and Energy Conversion, Xiangtan, 411100, China
}

\begin{abstract}
With the development of an intelligent microgrid transaction platform and blockchain technology, blockchain as a service (BaaS) on a cloud platform has received much attention. Currently, the problem of re-imaging the microgrid transaction mechanism is highlighted. Renewable energy must be consumed effectively and intelligently under the new transaction mechanism. To address these situations, this paper first builds the BaaS model, where blockchain technology is applied to the power transaction for intelligent distributed units. Then, a decision-making method is proposed for the intelligent unit to decide what it trades with. The method deals with transaction data in the cloud platform, obtains the probability distribution model of the microgrid transaction, and eventually achieves transaction decision making by using the Dempster Shafer (DS) evidence theory. The proposed transaction decision-making method provides more ways to reconstruct the microgrid transaction market and obtain effective management of market orders.
\end{abstract}

Keywords: intelligent distributed unit; BaaS; microgrid transaction market; DS evidence theory

(Submitted on May 3, 2018; Revised on June 2, 2018; Accepted on July 25, 2018)

(C) 2018 Totem Publisher, Inc. All rights reserved.

\section{Introduction}

Due to the current intermittency and distribution of renewable energy, the microgrid has received much attention for its convenience of consumption of nearby energy, its re-imagining energy transaction mode, and its reduced cost [1]. The traditional mechanism of power transactions requires frequent proofreading, and it is difficult to meet the stochastic change of the distributed energy price [2]. The prominent problem is that the transaction and utilization efficiency of renewable energy in a microgrid is low. The microgrid trading mechanism should have intelligence and flexibility, allowing users to make effective plans regarding the transfer and consumption of renewable energy [3-4].

Traditional power users are ordinary users and electricity institutions. However, this can also be extended to intelligent units for intelligent distributed energy resources. An intelligent unit includes not only the regular trading user, but also some intelligent power equipment of distributed energy resources. Among them, the original DG microgrid power interface inverter can upgrade to the advanced inverter, which follows the standard IEC 61850-90-7. The advanced inverter has the function of power quality regulation, which can control voltage and frequency autonomously. It allows the inverter system and other intelligent devices to assume more responsibilities in the process of the transaction [5]. Meanwhile, during the process of the transaction, the energy router has the function of communication and management, which can help realize the network interconnection of different intelligent units as well as the related detection process of the power exchange of the intelligent units [6].

In recent years, there has been much research on new virtualization energy storage and the new trading mechanism of the electricity market. Reference [7] proposes that using new cloud storage technology about distributed energy allows for flexibility in energy storage and release and reduces power price. It also builds a mathematical model to achieve the consumer of cloud storage at a low cost. The proposed method of cloud storage energy provides the possibility for microgrids to exchange virtualization energy. Reference [8] puts forward a kind of machine to machine (M2M) transaction

\footnotetext{
* Corresponding author.

E-mail address: xiaoxiangxiang_xtu@163.com
} 
form based on blockchain technology and introduces closely the technical details of blockchain in power transactions. The above references put forward specific trading technology and new forms of the power market. However, they do not involve special microgrids. To establish a set of intelligent microgrid transaction modes, it is necessary to build an information model of the microgrid market cloud platform. On the cloud platform, intelligent units can achieve a series of re-imaging forms of power trading behavior, such as cloud storage and cloud trading. Blockchain technology of no-center institution is introduced to build the power market of the machine to machine (M2M). It uses a high secrecy hash function to manage the data, which can greatly improve the confidentiality and non-tamperability of the transaction. On the other hand, the use of smart contracts in the transaction can further standardize the order of the transaction [9]. Reference [10] proposes using BaaS as the new technology for the Internet of things (IoT) and evaluates the performance of a cloud and edge hosted blockchain implementation. We can also adopt the BaaS for intelligent distributed energy resources. Intelligent units can use the trading data from the BaaS cloud platform to complete data processing and mining and then use the DS evidence theory to achieve the automated decision process about transaction object. Finally, it can then write smart contracts to realize it.

The first part of this paper gives the transaction cloud platform architecture based on the BaaS. The second part presents the specific process of electricity trading with smart contracts in the cloud platform. The third part introduces the basic theory of DS decision theory in decision making. The fourth part gives an example to prove the effectiveness of the trading decision making by software.

\section{Methodology}

The expected power generated by distributed generation is regarded as a virtual power resource and can be sold in the form of assets. The conventional microgrid transaction relies on central authority such as the power bureau, where the settlement time is long and the cost of manpower is higher. The establishment of a blockchain service platform can effectively solve the problems existing in the centralization structure, and the smart contract can manage the problems of default in an orderly way [11].

As shown in Figure 1, the blockchain as a service (BaaS) refers to a platform tool that is deployed on the cloud computing facilities and manages the blockchain network service. At present, many blockchain technology teams have distributed relevant BaaS platforms, which can be used to establish the microgrid trading cloud platform. The cloud platform of microgrid trading on BaaS provides the database for the decision of the transaction. The cloud platform contains Hardware-as-a-Service (IaaS), Platform-as-a-Service (PaaS), Software-as-a-Service (SaaS) and the client [12]. The IaaS layer is composed of the basic communication facilities, which construct the basic operating environment; the PaaS layer uses the platform bus to realize the registration, monitoring, and management status of the service; the SaaS layer covers the market information of four aspects, including power market transaction, market settlement, market service, and market analysis; and the client contains terminal devices that can contact others through the Internet to link all kinds of application services. The BaaS provides environmental support for decision making for microgrid virtual energy transaction.

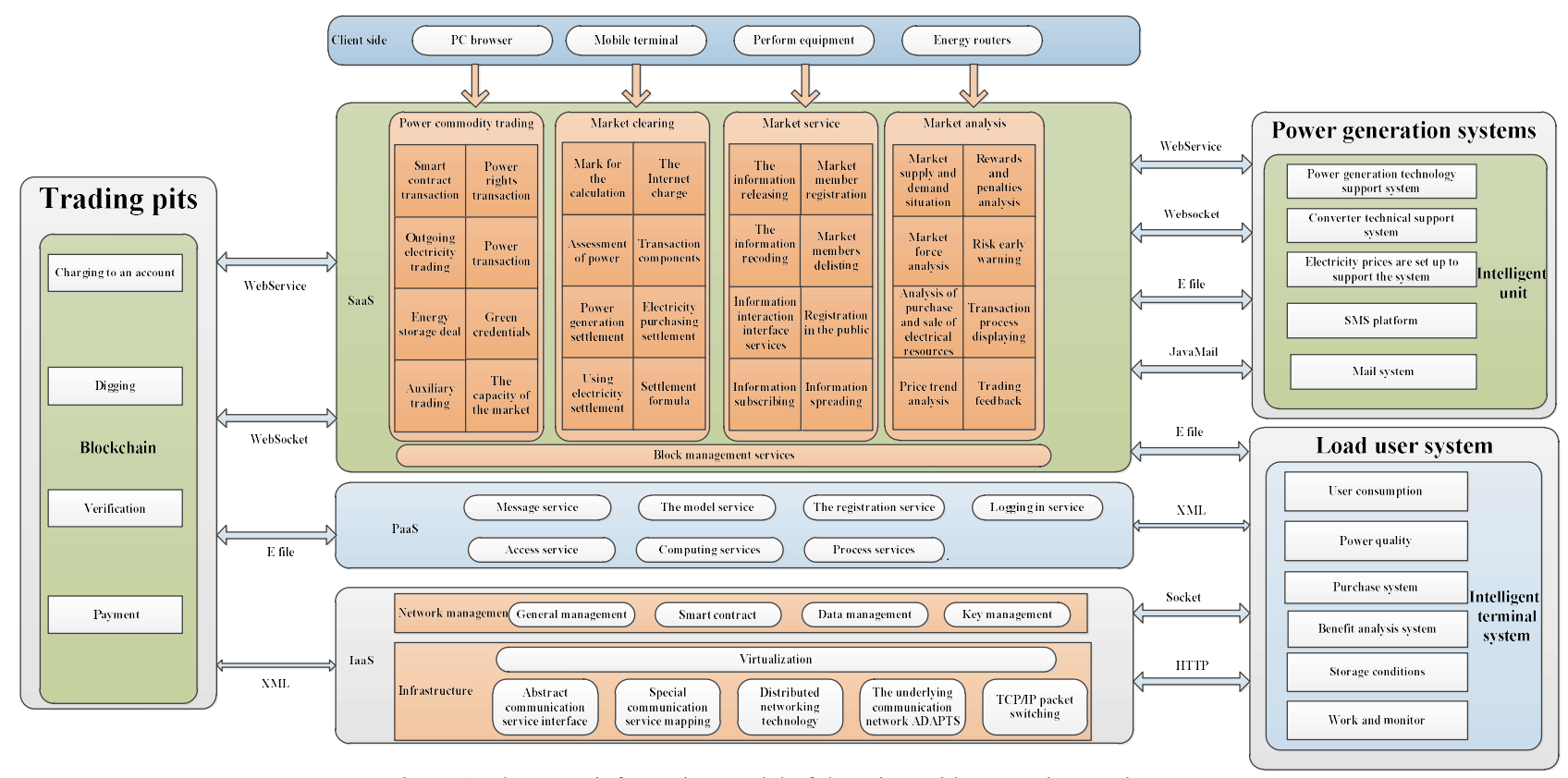

Figure 1. The BaaS information model of the microgrid transaction market 
In this paper, the power layer information is uploaded through the networked energy router system. Based on the Microsoft Azure cloud blockchain service, the intelligent units are registered with the power accounts online. The blockchain management interface can be quickly logged into through the browser. Each unit has a visual operating interface with an RPC interface address. The platform virtualizes the power resources and achieves the deployment and test of smart contracts.

\subsection{The Mainstream of Transaction Decision}

The decision of microgrid transaction based on BaaS is to make data mining under the data of historical transaction and complete the automatic transfer of electrical energy and value. The mainstream diagram of the transaction decision method on BaaS for the intelligent unit is described in Figure 2. The energy router is built for each intelligent unit, which is the intelligent interface of the energy Internet, and becomes an electric energy exchange link among different intelligent units. Firstly, it builds the BaaS for microgrid transaction. Then, the energy router uses energy flow detection to judge whether the internal power supply and demand is balanced. If it is balanced, the power quality situation awareness control is used. If it is not balanced, the evidence is excavated from the cloud platform containing much of the electricity transaction database, and the DS evidence theory is used to determine which one to unit trade with. After the decision, the content of the smart contract for transactions is determined and encoded. When all the intelligent units reach a consensus, the input and output of each transaction are determined. The next step is to judge whether the transaction is safe, not safe, or complete, and then it returns the contents of the redesign of the transaction contract. The network effectively connects the energy router and the BaaS platform to ensure the efficiency and real-time transaction among intelligent units in the microgrid.

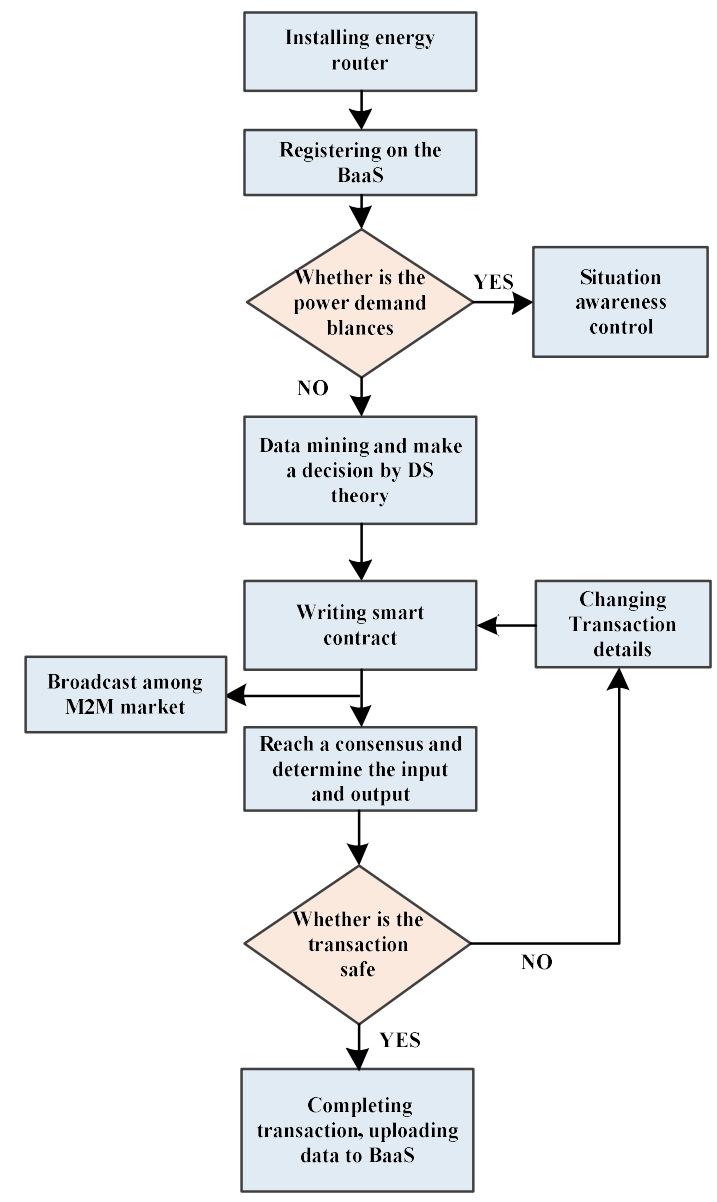

Figure 2. The mainstream diagram

\subsection{Implementation Scheme of BaaS Platform Transaction}

\subsubsection{The Trading Mechanism on BaaS}

Microgrid transactions need to be completed in the BaaS cloud platform, assuming that the kinds of intelligent distributed unit contain an advanced inverter system (AI), photovoltaic power generation unit (PV), wind power generation unit (WT), 
energy storage unit (ES), load shedding unit (IE), and circuit breaker controller (BC). Intelligent units manage the physical unit on the cloud platform and choose the corresponding working condition. The advanced inverter, following the definition of IEC 61850-90-7, can provide three modes of active power frequency operation mode, reactive voltage operation mode, and power generation mode for the power generation system to select an external operation mode. This means its benefits belong to the power generation unit [13].

The transaction process of intelligent units registered on the cloud platform is as follows: the smart contract that includes the transaction object and the quantity of the power is published by one who needs an expected power demand; the smart contract is transmitted to the regional main control node where the transaction object node is located firstly, and then it is transmitted to the corresponding energy router. The control system transmits the electrical energy, which meets the contract requirements on time. Then, it completes the transaction and packs the data to the blockchain.

As shown in Figure 3, energy routers detect whether the intelligent unit has energy flow. If there is no energy flow, the intelligent node can establish a microgrid Markov decision model (MDP), including the power quality requirement state model, the converter running model, the transfer probability matrix model, and the return matrix model. A microgrid is controlled by advanced converters to build a situation awareness model, predicting corresponding modes in advance and maintaining internal stability [5].

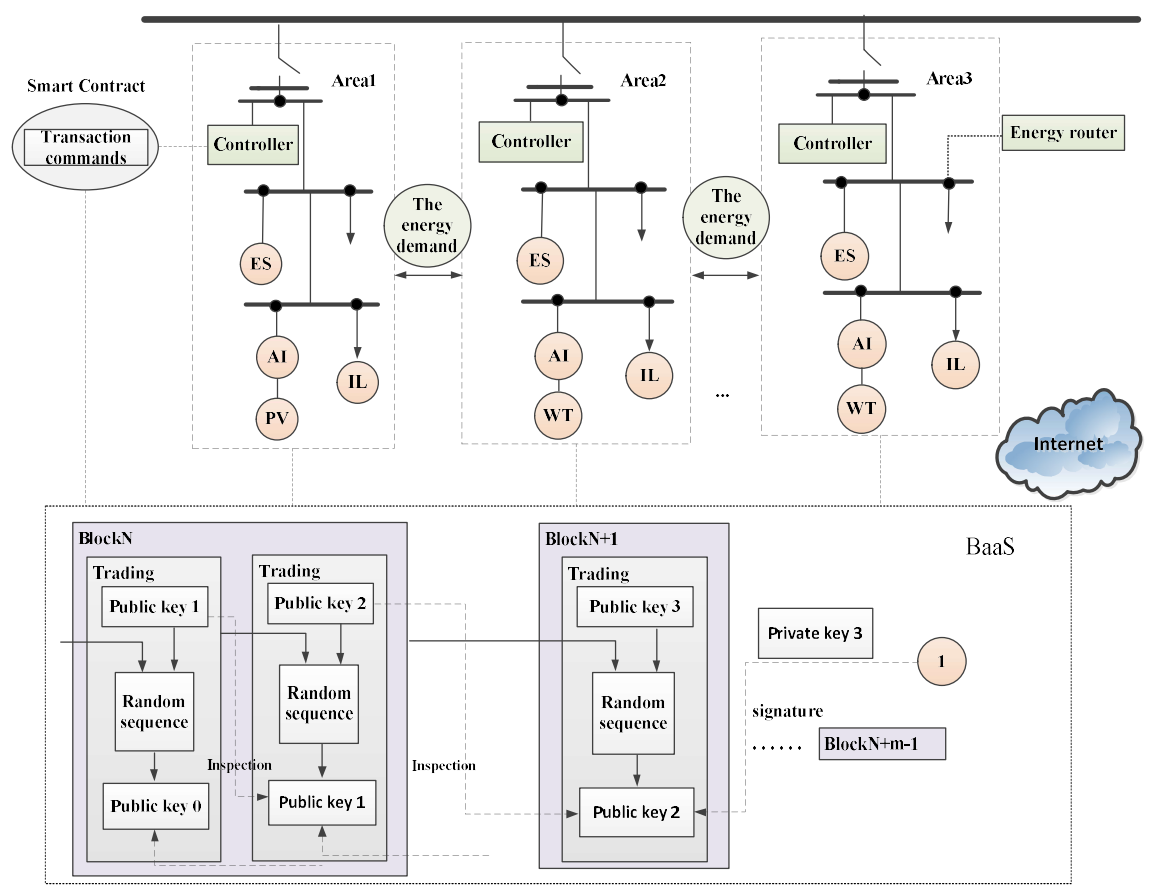

Figure 3. Physical framework of cloud trading platform

The energy routers can transfer the amount of power data to BaaS, which provides convenience for the user to manage energy transmission intelligently. The intelligent units register on the BaaS. The users can enter the visual interface and watch the situation of the transaction. The BaaS cloud platform provides an intelligent unit with a pair of public keys and private keys. The public key is the user's account address on the cloud platform, and the private key is the only key to operate the account.

If the energy router has detected the energy flow, the intelligent unit will generate transaction demand. The BaaS excavated the transaction information of the bus circuit breaker, advanced converter system, energy storage equipment, and resectable load transaction in the past time as the evidence source of the decision model, and it also used the D-S evidence theory to make a decision on which intelligent unit to trade with among a large number of intelligent units. After selecting good trading objects, the smart contract is published to BaaS and is set up including the transaction amount, the recipient address, and the public key. The participants sign respectively with their own private keys to ensure the validity and enforcement of the contract. The specific transaction security inspection mechanism can be determined by the power flow network constraint equation. If it is safe, during the time stipulated by the contract, both sides of the transaction will smoothly transfer the energy from the generator to the consuming party through the control of the energy router. 


\subsubsection{Determination of Potential Transaction Units}

The transaction basis of intelligent units is built on the power structure. It is essential to establish a power flow control network model for microgrid energy routers. In the power topology of a microgrid interconnected area, power flow achieves bidirectional flow.

It is assumed that the topology of the five intelligent units in the microgrid can be shown in the following diagram, where $\mathrm{S}$ represents the wind power unit and the remaining four units are power consumers.

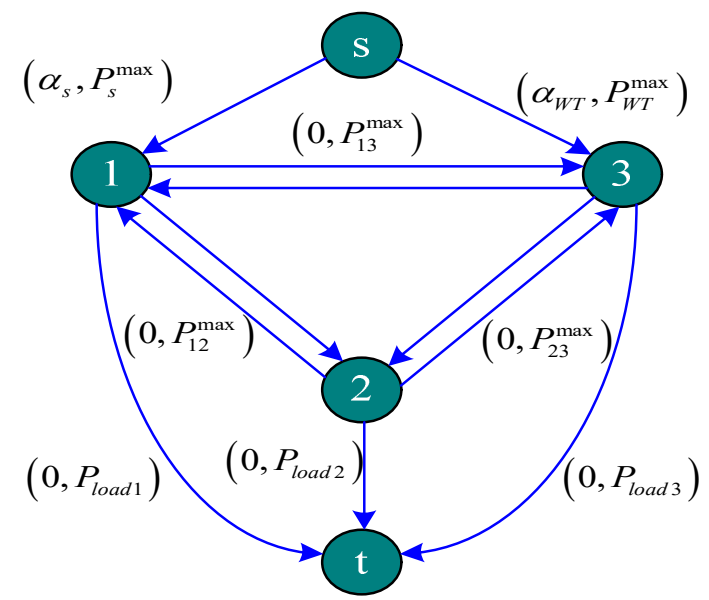

Figure 4. Microgrid topology model

For the topology graph, the power flow situation can be obtained, so that the power flow of the microgrid can be controlled by an energy router.

Usually, the power flow control of the microgrid can be described by the following objective function $F(x)$, which is described in Equation (1):

$$
F(x)=\min \left(\sum_{i \in S} \alpha_{i} \Delta P_{g i}+\sum_{i \in T} \beta_{i} \Delta P_{t i}+\sum_{i \in D} \gamma_{i} \Delta P_{l i}\right)
$$

Where $\Delta P_{g i} 、 \Delta P_{t i} 、 \Delta P_{l i}$ are changes in power generation, transmission, and load, respectively, and $\alpha_{i} 、 \beta_{i} 、 \gamma_{i}$ are the risk coefficients. $S, D$, and $T$ represent the collection of power, transmission line, and demand area, respectively. The objective function is the total cost of transmission of electric energy from power generation to load side.

Equation (2) represents the power balance condition.

$$
\sum_{i \in S}^{s . t} \Delta P_{g i}=\sum_{i \in T} \Delta P_{l o s s i}+\sum_{i \in D} \Delta P_{l i}
$$

Equation (3) represents physical operating limits.

$$
P_{t i}+\Delta P_{l i} \leq P_{t i \max }
$$

With the distributed theory, the graph theory can solve this optimization problem. The distributed method is based on an energy router system to control energy flow. The feasible path can be found between the smart units of the microgrid through the main control panel of the energy router, in order to achieve the purpose of controlling power flow.

The unit that passes through the feasible path is the potential transaction unit. Among potential transaction units, the DS evidence mining theory is used to select the optimal transaction unit, and then the smart contract is formulated. 


\subsubsection{Smart Contract}

Unlike the usual trading environment, the randomness and intermittency of the distributed power production are more prominent. On the BaaS, a smart contract is generated during the transaction, and it can manage the transaction order compulsively.

The smart contract is a code program on the distributed ledger that aims at completing the logical settings required by automated exchanges. Both sides of the transaction transfer electronic wallet funds by the corresponding content of the contract, the transaction generates a random sequence by the SHA algorithm, and the data preservation is encrypted.

Taking the actual situation as an example, after the decision of the intelligent object is selected by the D-S evidence theory, the intelligent unit A needs to trade with the intelligent unit B. A is a photovoltaic power unit while B is a power consumption unit, and A is expected to send $10 \mathrm{KWh}$ to $\mathrm{B}$ in a certain period by smart contract content. In reality, due to the weather change, A produces only $8 \mathrm{KWh}$; it needs additional conditions in the smart contract. If the power generation does not reach the predetermined condition, A will reduce the price to deal with $\mathrm{B}$, and the specific details are encoded into the smart contract.

\subsubsection{Safety Inspection}

To consider the power security under the microgrid transaction environment, we need to consider the power flow.

There are two types of power flow calculation in the microgrid environment, namely, the power flow calculation with a balance node and the power flow calculation without a balance node [14]. The unified power flow equation based on nodal power is an applicable method for the two types of power flow calculation. The unified power flow method of a microgrid is described by Equation (4):

$$
F(x)=0, x \in R^{n}
$$

Where $x$ represents the n-dimensional vector of the independent variable in the power system. The results of power flow calculations can be considered as a set of nonlinear equations.

To apply the particularity of the new energy power generation, some of the distributed generation (DG) nodes adopt the droop control mode, which can be called the power droop node (PD). As the carrier of information exchange between different droop control nodes, the frequency is an unknown system variable. The number of all nodes are $\mathrm{N}$ in the microgrid without a balance node, where the number of PQ nodes are $\mathrm{M}_{1},\left(1,2, \cdots, \mathrm{M}_{1}\right)$, the number of PD nodes are $\mathrm{M}_{2},\left(\mathrm{M}_{1}+1, \cdots\right.$, $\left.\mathrm{M}_{1}+\mathrm{M}_{2}\right)$, and the remaining nodes are PV nodes $\left(\mathrm{M}_{1}+\mathrm{M}_{2}+1, \cdots, N\right)$. When the active and the reactive power of droop nodes reach their maximum capacity, the PD node can convert into the PQ node. The nonlinear nodal power equations for the power flow calculation of the microgrid are:

$$
\left\{\begin{array}{l}
F_{P P Q 1}\left(f, U_{1}, \cdots, U_{\left(M_{1}+1\right)}, \cdots, U_{\left(M_{1}+M_{2}\right)}, \delta_{2}, \cdots, \delta_{M_{1}}, \delta_{M_{1}+1}, \cdots, \delta_{M_{1}+M_{2}}, \cdots, \delta_{N}\right)=0 \\
\cdots \\
F_{P P Q M_{1}}\left(f, U_{1}, \cdots, U_{\left(M_{1}+1\right)}, \cdots, U_{\left(M_{1}+M_{2}\right)}, \delta_{2}, \cdots, \delta_{M_{1}}, \delta_{M_{1}+1}, \cdots, \delta_{M_{1}+M_{2}}, \cdots, \delta_{N}\right)=0 \\
F_{P D\left(M_{1}+1\right)}\left(f, U_{1}, \cdots, U_{\left(M_{1}+1\right)}, \cdots, U_{\left(M_{1}+M_{2}\right)}, \delta_{2}, \cdots, \delta_{M_{1}}, \delta_{M_{1}+1}, \cdots, \delta_{M_{1}+M_{2}}, \cdots, \delta_{N}\right)=0 \\
\cdots \\
F_{P D\left(M_{1}+M_{2}\right)}\left(f, U_{1}, \cdots, U_{\left(M_{1}+1\right)}, \cdots, U_{\left(M_{1}+M_{2}\right)}, \delta_{2}, \cdots, \delta_{M_{1}}, \delta_{M_{1}+1}, \cdots, \delta_{M_{1}+M_{2}}, \cdots, \delta_{N}\right)=0 \\
F_{P P V\left(M_{1}+M_{2}+1\right)}\left(f, U_{1}, \cdots, U_{\left(M_{1}+1\right)}, \cdots, U_{\left(M_{1}+M_{2}\right)}, \delta_{2}, \cdots, \delta_{M_{1}}, \delta_{M_{1}+1}, \cdots, \delta_{M_{1}+M_{2}}, \cdots, \delta_{N}\right)=0 \\
\cdots \\
F_{P P V N}\left(f, U_{1}, \cdots, U_{\left(M_{1}+1\right)}, \cdots, U_{\left(M_{1}+M_{2}\right)}, \delta_{2}, \cdots, \delta_{M_{1}}, \delta_{M_{1}+1}, \cdots, \delta_{M_{1}+M_{2}}, \cdots, \delta_{N}\right)=0
\end{array}\right\}
$$

Use the improved LMTR power flow algorithm to obtain the corresponding Jacobi matrix [15]. It is as follows: 


$$
J_{k}=\left[\begin{array}{ccc}
A_{M_{1} \times\left(M_{1}+M_{2}\right)}^{\prime} & B_{M_{1} \times(N-1)} & E_{M_{1} \times 1} \\
A_{M_{2} \times\left(M_{1}+M_{2}\right)}^{\prime} & B_{M_{2} \times(N-1)} & F_{M_{2} \times 1} \\
A_{\left(N-M_{1}-M_{2}\right) \times\left(M_{1}+M_{2}\right)}^{\prime} & B_{\left(N-M_{1}-M_{2}\right) \times(N-1)} & E_{\left(N-M_{1}-M_{2}\right) \times 1} \\
C^{\prime}{ }_{M_{1} \times\left(M_{1}+M_{2}\right)} & D_{M_{1} \times(N-1)} & G_{M_{1} \times 1} \\
C^{\prime \prime}{ }_{M_{2} \times\left(M_{1}+M_{2}\right)} & D_{M_{2} \times(N-1)} & G_{M_{2} \times 1}
\end{array}\right]
$$

Each block matrix is shown in Equation (5).

$$
\begin{aligned}
& A_{i j}^{\prime}=\frac{\partial P_{i}}{\partial U_{j}}+\frac{\partial P_{L i}}{\partial U_{j}}, B_{i j}=\frac{\partial P_{i}}{\partial \phi_{j}}, C^{\prime}{ }_{i j}=\frac{\partial Q_{i}}{\partial U_{j}}+\frac{\partial Q_{L i}}{\partial U_{j}}, D_{i j}=\frac{\partial Q_{i}}{\partial \phi_{j}}, \\
& E_{i j}=\frac{\partial P_{L i}}{\partial f}, F_{i j}=\frac{\partial P_{L i}}{\partial f}+\frac{\partial P_{G i}}{\partial f}, G_{i j}=\frac{\partial Q_{L i}}{\partial f}, C^{\prime \prime}{ }_{i j}=\frac{\partial Q_{i}}{\partial U_{j}}+\frac{\partial Q_{L i}}{\partial U_{j}}+\frac{\partial Q_{G i}}{\partial U_{j}}
\end{aligned}
$$

$P_{L i}$ and $P_{G i}$ are the node load power and the power supply, respectively.

According to the conditions of the transaction and related prediction, we get the power distribution after assuming the transaction is successful. The qualification range of the steady state frequency is $[0.994,1.006]$ pu at normal operation, the qualified range of the node voltage amplitude is $[0.9400,1.0600] \mathrm{pu}$, and the upper limit of the power line is $0.53 \mathrm{pu}$. If the above conditions are satisfied, the transaction meets the power constraint conditions.

\subsection{Transaction Decision Model}

The key problem is how to choose the most suitable unit as the trading object among many potential transaction units that meet the limit of the above power conditions and the restricted conditions of the transaction security. The decision fusion theory can greatly improve the rationality of the decision in selecting the transaction object. Furthermore, it can make the decision through the fusion of the complementary evidence theory, which is the Dempster Shafer (DS) evidence theory.

\subsubsection{DS Evidence Theory}

Under the premise of security detection, the introduction of the centralized distributed account ledger makes the transaction data of intelligent units complex and highly random, and there are various uncertain mathematical reasoning problems in the cloud platform field. The DS evidence theory is introduced to decide whether intelligent units participate in transactions [16].

For the universal set $\Omega$, the power set is $2^{\Omega}$. A probability assignment function $M: 2^{\Omega} \rightarrow[0,1]$ satisfies Equation (6):

$$
M(A)=c^{-1} \sum_{A_{1} \cap A_{2}=A} M_{1}\left(A_{1}\right) M_{2}\left(A_{2}\right), A \neq \Phi
$$

Let $M_{1}$ and $M_{2}$ be two probability distribution functions on the same power set $2^{\Omega}$, and then an orthogonal sum function $M_{1}+M_{2}$ can be obtained as Equation (7):

$$
M(A)=c^{-1} \sum_{A_{1} \cap A_{2}=A} M_{1}\left(A_{1}\right) M_{2}\left(A_{2}\right), A \neq \Phi
$$

Where $c=1-\sum_{A_{1} \cap A_{2}=\Phi} M_{1}\left(A_{1}\right) M_{2}\left(A_{2}\right)=\sum_{A_{1} \cap A_{2} \neq \Phi} M_{1}\left(A_{1}\right) M_{2}\left(A_{2}\right)$.

Equation (6) to general $M=M_{1}+M_{2}+\cdots+M_{n}$ is defined as Equation (8):

$$
M(A)=c^{-1} \sum_{\cap A_{i}=A} \prod_{1 \leq i \leq n} M_{\mathrm{i}}\left(A_{i}\right), A \neq \Phi
$$


Where $c=1-\sum_{\cap} \prod_{A_{i}=\Phi} M_{\mathrm{i}}\left(A_{i}\right)=\sum_{\cap A_{i}=\Phi} \prod_{1 \leq i \leq n} M_{\mathrm{i}}\left(A_{i}\right)$

\subsubsection{Decision-Making Model}

In the decision-making process, the transaction situation among distributed accounting ledgers of power generation forecast, line fault probability, storage device available probability, and the probability of load excision in a certain period of time can be regarded as sources of evidence.

As shown in Figure 5, for any intelligent unit, the probability distribution of receiving a transaction contract from another intelligent unit is obtained by establishing the probability model. The DS evidence theory is used to obtain a new proposition and the corresponding confidence fusion. The mathematical model of transaction decisions for the intelligent unit in a certain period is shown in Equation (9):

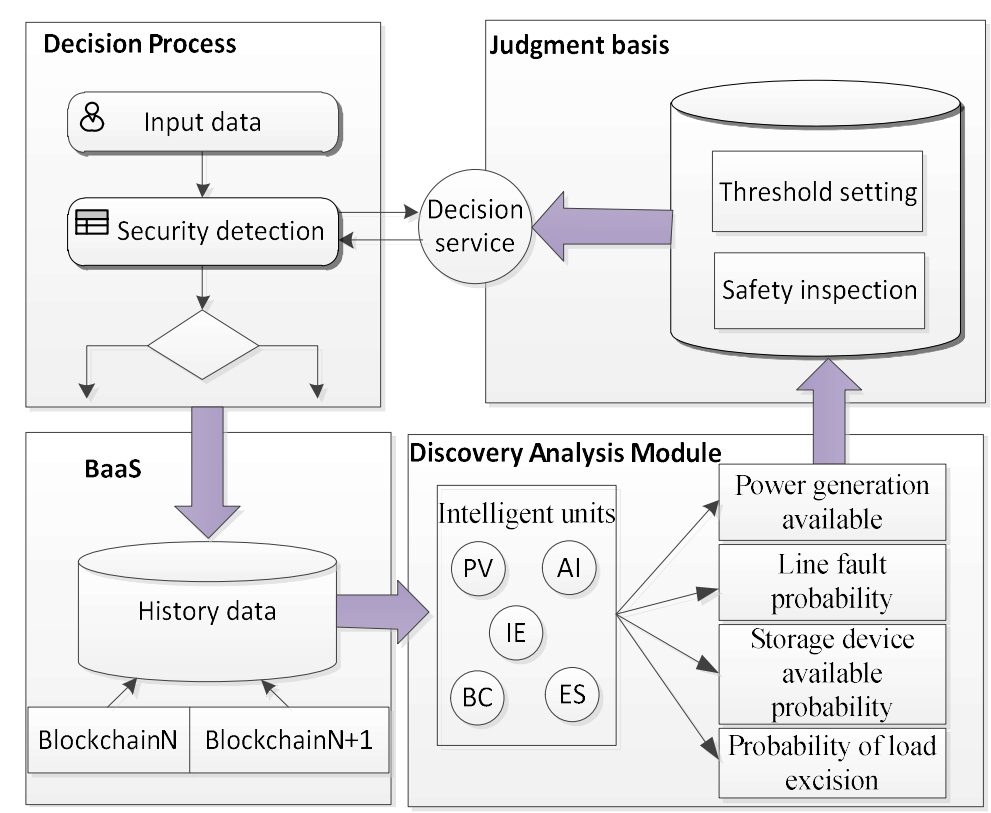

Figure 5. The decision management cycle of security

If $x, y \subset \Omega$, it meets $M(x)=\max \left\{M\left(x_{i}\right), x_{i} \subset \Omega\right\}, M(\mathrm{y})=\max \left\{M\left(y_{i}\right), y_{i} \subset \Omega \& x \neq y\right\}$,

$$
\left\{\begin{array}{l}
M(x)-M(y)>\varepsilon_{1} \\
M(\Omega)<\varepsilon_{2} \\
M(x)>M(\Omega)
\end{array}\right.
$$

Where $x$ is the final judgment value and $\varepsilon_{1}$ and $\varepsilon_{2}$ are the preset threshold values.

The trader chooses the biggest final judgment among the potential objects as the transaction object, and then it encodes the smart contract.

\section{Case Study}

In this paper, a smart grid transaction model with many intelligent units is built, including a wind power unit regarded as A. Building a BaaS platform on the Azure cloud computing platform, the Azure cloud computing platform provides users with open IaaS and PaaS services, deploying a blockchain transaction network in the cloud based on the topology of the microgrid and the characteristics of the transaction. 
The Azure cloud computing platform provides a high-reliability environment for blockchain transactions. Compare the services under different blockchain environments for the probability reliability. According to historical experience values, the test results are shown for the simple environment, the ordinary single point environment, the common cluster environment, and the BaaS cloud platform environment.

Table 1 shows that the BaaS cloud platform is more reliable than other trading environments and allows for longer working time each year to meet the environmental requirements of microgrid transactions.

Table 1 . The probability reliability

\begin{tabular}{|l|c|c|}
\hline \multicolumn{1}{|c|}{ Typical environment } & Time not available & Probability reliability \\
\hline Simple environment & 1.2 months & $90 \%$ \\
\hline Ordinary single point environment & 3.6 days & $99 \%$ \\
\hline Common cluster environment & 8.6 hours & $99.9 \%$ \\
\hline BaaS & 51.6 minutes & $99.99 \%$ \\
\hline
\end{tabular}

A transaction database is derived from the Azure cloud computing platform. The program is compiled in MATLAB, and $\mathrm{A}$ is used as the trading party to obtain the distribution of the final judgment $\mathrm{x}$ of the intelligent units trading with the $\mathrm{A}$. In Figure 6, the blue star represents the $x$ of the intelligent units in the transaction with load shedding, the green rhombus represents the transaction with storage equipment, and the red circle represents the transaction with an advanced converter system. The $\mathrm{x}$-value and $\mathrm{y}$-value are optimal judgments of different variance values.

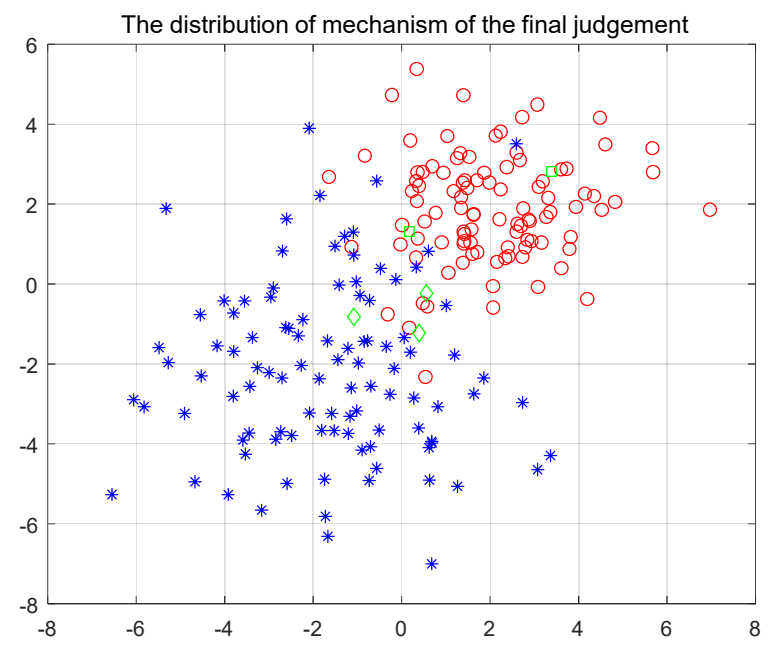

Figure 6. The distribution of mechanism of the final judgment

According to the final judgment based on the volume distribution, the transaction object can be determined, and we will carry out the smart contract code. This is shown by the following example:

$>$ myContract $=$ MyContract.new $(\{$ from:address, data:contract.code $\})$

\{

abi: $[\{$

constant: false,

inputs: \{

name: "a",

type: "uint256"

\}],

$$
\}
$$

address: undefined,

transactionHash: "0xd10602e2099ab5873c762f070eb90a9fd559270484fbcebd4170d441848b9232"

\} 
On the platform, the two units discuss the scheduled completion date and the quantity of the transaction based on the actual conditions, and they add restrictions to the internal programming of the smart contract. The results displayed in the platform show that $\mathrm{A}$ is forced to receive some punishments due to the random power output of the new energy generation. It is as follows:

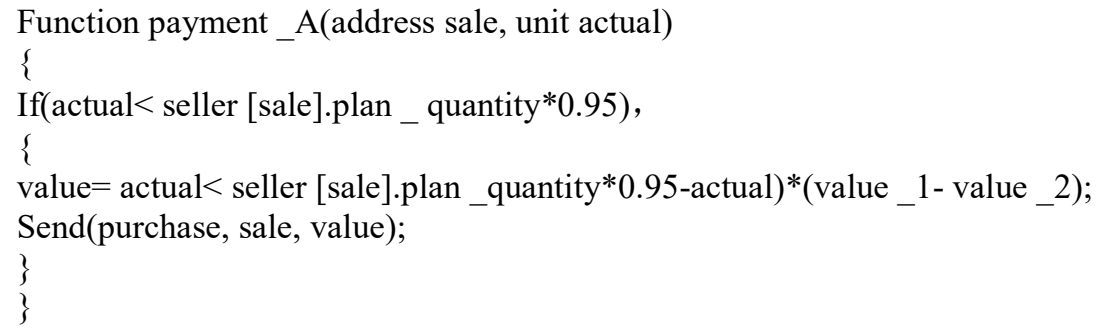

After the asset transfer is successful, the transaction data is stored in the Merkle tree on the BaaS, and the deal is completed.

\section{Conclusion}

Due to the conventional transaction mode of microgrids, it is difficult to greatly enhance the efficiency of the transfer and utilization of renewable energy. The traditional electricity trading model is no longer superior. Utilizing a microgrid cloud transaction platform based on BaaS and DS evidence theory, this paper proposes a transaction decision method for the distributed energy resource system and builds the transaction mechanism and automatic decision model. It provides more ways to improve the utilization efficiency of distributed energy and manage the order of the microgrid transaction. Next, we will build a specific energy internet that contains a large number of energy router platforms for blockchain trading to actually operate and observe its effects.

\section{Acknowledgment}

The authors would like to thank the Collaborative Innovation Center of Wind Power Equipment and Energy of China for their support.

\section{References}

1. G. Zhang, C. W. Jiang, X. Wang, B. S. Li, and H. G. Zhu, "Bidding Strategy Analysis of Virtual Power Plant Considering Demand Response and Uncertainty of Renewable Energy," IET Generation, Transmission \& Distribution, Vol. 11, No. 13, pp. 3268-3277, 2017

2. S. Malla and K. Christensen, "Reducing Power Use and Enabling Oversubscription in Multi-tenant Data Centers using Local Price," in Proceedings of 2017 IEEE International Conference on Autonomic Computing (ICAC), pp. 161-166, Columbus, OH, 2017

3. Z. Li, "A Survey on Modeling Energy Consumption of Cloud Applications: Deconstruction, State of the Art, and Trade-Off Debates," IEEE Transactions on Sustainable Computing, Vol. 2, No. 3, pp. 255-274, 2017

4. G. Ozcan, M. C. Gursoy, and J. Tang, "Spectral and Energy Efficiency in Cognitive Radio Systems with Unslotted Primary Users and Sensing Uncertainty," IEEE Transactions on Communications, Vol. 65, No. 10, pp. 4138-4151, 2017

5. M. J. Chen, B. Duan, and Z. Yang, "State Machine of Advanced Inverter: An Approach to Situation Awareness and Proactive Operation," in Proceedings of 2016 IEEE Power and Energy Society General Meeting (PESGM), pp. 1-5, Boston, MA, 2016

6. P. H. Nguyen, W. L. Kling, and P. F. Ribeiro, "Smart Power Router: A Flexible Agent-based Converter Interface in Active Distribution Networks," IEEE Transactions on Smart Grid, Vol. 2, No. 3, pp. 487-495, 2011

7. J. Liu, N. Zhang, C. Kang, D. S. Kirschen, and Q. Xia, "Decision-Making Models for the Participants in Cloud Energy Storage," IEEE Transactions on Smart Grid, Vol. PP, No. 99, pp. 1, 2017

8. J. J. Sikorski, J. Haughton, and M. Kraft, "Blockchain Technology in the Chemical Industry: Machine-to-machine Electricity Market,” Applied Energy, Vol. 195, pp. 234-246, 2017

9. L. Thomas, C. Long, and P. Burnap, "Automation of the Supplier Role in the GB Power System using Blockchain-based Smart Contracts," CIRED - Open Access Proceedings Journal, Vol. 2017, No. 1, pp. 2619-2623, 2017

10. M. Samaniego and R. Deters, "Blockchain as a Service for IoT," in Proceedings of 2016 IEEE Green Computing and Communications (GreenCom) and IEEE Cyber, Physical and Social Computing (CPSCom) and IEEE Smart Data (SmartData), pp. 433-436, Chengdu, 2016

11. X. Tai, H. Sun, and Q. Guo, "Electricity Transactions and Congestion Management based on Blockchain in Energy Internet," Power System Technology, Vol. 40, No. 12, pp. 3630-3638, 2016

12. L. Shi, P. Shao, and X. Zhang, "Discussion on Architecture of New-generation Electricity Trading Platform," Automation of 
Electric Power System, Vol. 41, No. 24, pp. 67-76, 2017

13. P. K. Sharma, M. Y. Chen, and J. H. Park, "A Software Defined Fog Node based Distributed Blockchain Cloud Architecture for IoT," IEEE Access, Vol. PP, No. 99, pp. 1-1, 2017

14. H. Peng, Y. Cao, and X. Huang, "Continuation Power Flow Calculation of Microgrid without Balance Node," Automation of Electric Power Systems, Vol. 4, pp. 6-10, 2016

15. H. Peng, Y. Cao, and X. Huang, "Power Flow Calculation of Islanded Microgrids based on BFGS Trust Region Method," Proceedings of the CSEE, Vol. 34, No. 16, pp. 2629-2638, 2014

16. G. Dong and G. Kuang, "Target Recognition via Information Aggregation Through Dempster-Shafer's Evidence Theory," IEEE Geoscience and Remote Sensing Letters, Vol. 12, No. 6, pp. 1247-1251, 2015

Xiangxiang Xiao graduated from the North China Institute of Science and Technology and is currently studying at Xiangtan University, where he is a Master's student of Electrical Engineering. His main research interests are microgrids and blockchain.

Bin Duan is a professor and doctoral supervisor of Xiangtan University. He is a member of the Master's degree in Electronics and Communication Engineering at Xiangtan University, a senior member of the China Electrotechnical Society in Xiangtan City, and a second level candidate of the Hunan Province 121 talent project.

Jun Lai graduated from the Hunan Institute of Technology and is currently a Master's student at Xiangtan University. His main research interest is wind power information security.

Tao Li graduated from the Hunan Institute of Engineering and is currently a Master's student at Xiangtan University studying Electrical Engineering. His main research interests are blockchain and artificial intelligence. 